\title{
Significance of G Test [(1-3)- $\beta$-D-Glucan Assay] in the Diagnosis and Guidance of Clinical Medication of Invasive Fungal Infection
}

\author{
Lianfang Xue ${ }^{1}$, Suishan Qiu ${ }^{1}$, Guangchao Yu ${ }^{2}$, Qin-Ai Zhu ${ }^{2}$, Shasha Li ${ }^{1}$, Hui Liu ${ }^{1}$, Yuping Wang ${ }^{1}$, \\ Fengtian Ouyang ${ }^{3}$, Wenyu Gong ${ }^{4}$, * \\ ${ }^{1}$ Department of Pharmacy, The First Affiliated Hospital of Jinan University, Guangzhou, China \\ ${ }^{2}$ Department of Laboratory, The First Affiliated Hospital of Jinan University, Guangzhou, China \\ ${ }^{3}$ College of Pharmacy, Jinan University, Guangzhou, China \\ ${ }^{4}$ Department of Nephrology, The First Affiliated Hospital of Jinan University, Guangzhou, China
}

Email address:

gongwenyugz@qq.com (Wenyu Gong)

${ }^{*}$ Corresponding author

\section{To cite this article:}

Lianfang Xue, Suishan Qiu, Guangchao Yu, Qin-Ai Zhu, Shasha Li, Hui Liu, Yuping Wang, Fengtian Ouyang, Wenyu Gong. Significance of G Test [(1-3)- $\beta$-D-Glucan Assay] in the Diagnosis and Guidance of Clinical Medication of Invasive Fungal Infection. American Journal of Internal Medicine. Vol. 7, No. 2, 2019, pp. 46-50. doi: 10.11648/j.ajim.20190702.15

Received: April 13, 2019; Accepted: May 21, 2019; Published: June 11, 2019

\begin{abstract}
Objective: G test [(1-3) - $\beta$-D-glucan assay] was a novel texting method for invasive fungal infection (IFI). The study evaluated the diagnostic value of $G$ test for IFI by comparing $G$ test with fungal culture method, and analyze the guiding significance of $\mathrm{G}$ test and fungal culture in clinical medication. Methods: 373 inpatients with suspected IFI in the first affiliated hospital of Jinan university from January to December 2017 were retrospectively analyzed. G test and fungal culture results were collected, and their positive rate, sensitivity, specificity, positive and negative predictive values were calculated. The value and rationality of $\mathrm{G}$ test for clinical diagnosis of IFI was evaluated by taking the relief and cure of infection symptoms of patients as clinical effective criteria. Results: Among 373 suspected IFI patients, the positive rate of G experiment was $39.95 \%$, higher than that of fungus culture $(28.95 \%, \mathrm{P}<0.001)$. The positive rate of combined $\mathrm{G}$ test and fungal culture was $43.70 \%$. To evaluate the consistency of $\mathrm{G}$ test with fungal culture method, $\kappa$ value was $0.596(\mathrm{P}<0.001)$. When positive fungal culture was used as the criterion for the diagnosis of IFI, the sensitivity, specificity, positive predictive value and negative predictive value of $\mathrm{G}$ test for the diagnosis of IFI were $87.04 \%, 79.25 \%, 63.09 \%$ and $93.75 \%$, respectively. All 373 patients with IFI received antifungal therapy, and the effective rate of the both methods positive group was $72.34 \%$, higher than $42.86 \%$ in the only fungal culture positive group $(\mathrm{P}=0.033)$ and $30.48 \%$ in the both methods negative group $(\mathrm{P}<0.001)$. The effective rate of the only $\mathrm{G}$ test positive group was $58.18 \%$, higher than that of the both methods negative group $(30.48 \%, \mathrm{P}<0.001)$. There was no significant difference in the therapeutic efficiency between the only $\mathrm{G}$ test positive group and the only fungal culture positive group ( $\mathrm{P}=0.303$ ). Conclusion: G test was an effective diagnostic method of IFI, and combined with fungal culture could improve its positive rate and have a higher guiding value for clinical medication.
\end{abstract}

Keywords: Invasive Fungal Infection, G Test, (1-3)- $\beta$-D-Glucan, Diagnostic Value, Fungal Culture

\section{Introduction}

With extensive solid organ and hematopoietic stem cell transplantation, as well as the extensive use of corticosteroids, broad-spectrum antibiotics, tumor chemotherapy drugs and many kinds of catheter intervention, the prevalence of invasive fungal infections (IFI) sharply increased [1], which was a common cause of morbidity and mortality of immunocompromised patients. Since the clinical manifestation of IFI was nonspecific and clinical diagnosis was very difficult, it could easily lead to misdiagnosis and missed diagnosis, resulting in the increased mortality in severe 
patients $[1,2]$.

At present, etiological examination (especially fungal culture) was still a routine diagnostic standard for IFI, which could identify the fungal species causing infection and provided basis for clinical rational use of antifungal drugs according to in vitro drug sensitivity test. Fungal culture had the disadvantages of long time and low success rate of culture, easy to be interfered by the external environment and high rate of missed detection. Therefore, it is difficult to achieve the goal of early diagnosis, early treatment and reduction of mortality by traditional pathogenic examination. However, the key to IFI lay in early diagnosis and early intervention [3].

Glucan was widely found in fungal cell walls, accounting for $80 \% \sim 90 \%$ of its dry weight, among which (1-3)- $\beta$-D-glucan accounted for more than $50 \%$ of the components in fungal wall. Previous studies had shown that all fungal cell membranes except zygomycetes and cryptococcus contained (1-3)- $\beta$-D-glucan, while cell components and extracellular fluids of other microorganisms, viruses, animals and humans did not. Therefore, (1-3)- $\beta$-D-glucan assay (so called $\mathrm{G}$ test) in body fluids was an important basis for the diagnosis of IFI [4]. The concentration of (1-3)- $\beta$-D-glucan was very low in normal people, but it could be significantly increased in IFI, so G test could effectively predict early IFI [5].

Therefore, this study will explore the clinical value of $\mathrm{G}$ test in early diagnosis of IFI by comparing the results of $\mathrm{G}$ test and fungal culture in suspected IFI patients, and analyze the guiding significance of $G$ test and fungal culture in clinical medication.

\section{Method}

\subsection{Study Population}

A total of 373 suspected IFI patients were collected from the first affiliated hospital of Jinan university from January to December, 2017, with an average age of 48 years, including 211 males and 162 females. Inclusion criteria: 1. Patients met the diagnostic criteria for suspected IFI according to the clinical symptoms and signs, imaging and microbiological test results and the use of antifungal drugs and efficacy [6, 7]. 2 . Both $\mathrm{G}$ test and fungal culture were conducted. The exclusion criteria were: lack of $\mathrm{G}$ test or fungal culture results; lack of clinical outcome data and mental abnormalities cannot cooperate.

\subsection{Collect Data}

The results of $\mathrm{G}$ test and fungal culture, the medication and therapeutic effect of antifungal therapy were collected. The cases were retrospectively analyzed and classified as effective (including cure and improvement) and ineffective according to the clinical outcomes of the patients. (1) Effective meant normal body temperature, significantly improved symptoms and signs, and normal inflammatory indicators; (2) ineffective meant no obvious improvement in the condition.

\subsection{G Test [(1-3)-B-D-Glucan Assay]}

Mb-80 microorganism rapid dynamic detection system (Tianjin Danna Company) and fungi (1-3)- $\beta$-D-glucan quantitative assay kit (Tianjin Danna Company) were used for dynamic photometric detection. (1-3)- $\beta$-D-glucan concentration $\geq 95 \mathrm{pg} / \mathrm{ml}$ was positive, and (1-3)- $\beta$-D-glucan concentration $<95 \mathrm{pg} / \mathrm{ml}$ was negative.

\subsection{Fungal Culture}

Automatic microbial identification and drug sensitivity analysis system and related reagents were used (BioMerieux, France).

\subsection{Statistical Analysis}

Categorical variables were expressed as the frequency and percentage. Differences between categorical variables were analyzed using a chi-square test or double-tailed Fisher's exact test, depending on applicability. $\kappa$ value was used to evaluate the consistency of $\mathrm{G}$ test and fungal culture results.

All values are two-tailed, and $P<0.05$ was considered statistically significant. Data were analyzed using IBM SPSS Statistics version 25.0 for Windows (IBM, Armonk, NY, USA).

\section{Result}

\subsection{G Test}

Among the 373 suspected IFI patients, 149 (39.95\%) were positive for $\mathrm{G}$ test.

\subsection{Fungal Culture}

108 patients $(28.95 \%)$ were positive for fungal culture. There were 60 cases of Candida albicans $(55.55 \%), 21$ cases of Candida tropicalis (19.44\%), 11 cases of Candida glabrata (10.19\%) and 6 cases of candida parapsilokis (5.56\%) (Figure 1).

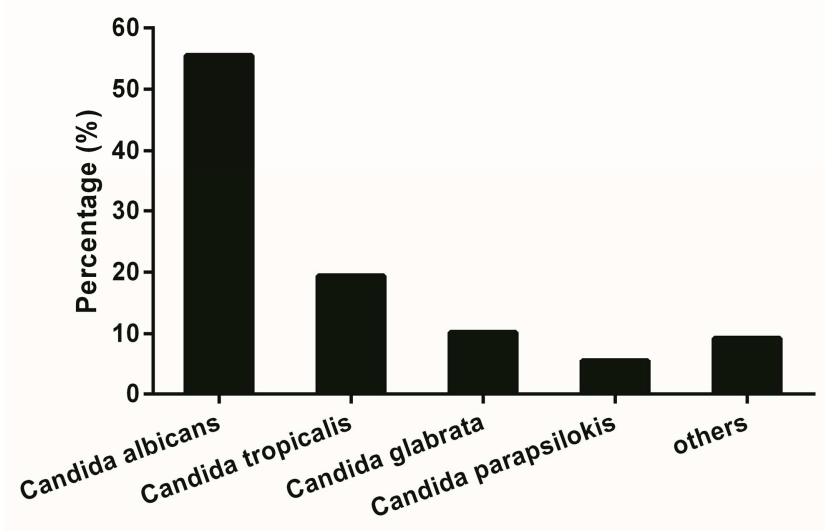

Figure 1. Fungal distributions of IFI.

The sample distribution of 108 positive culture cases was derived from 14 cases of blood (12.96\%), 52 cases of sputum 
(48.15\%), 14 cases of urine $(12.96 \%), 6$ cases of feces $(5.56 \%)$, 10 cases of ascites $(9.26 \%), 5$ cases of peritoneal dialysis fluid (4.63\%) and 7 cases of other types $(6.48 \%)$ including catheter secretions and vaginal leucorrhea (Figure 2).

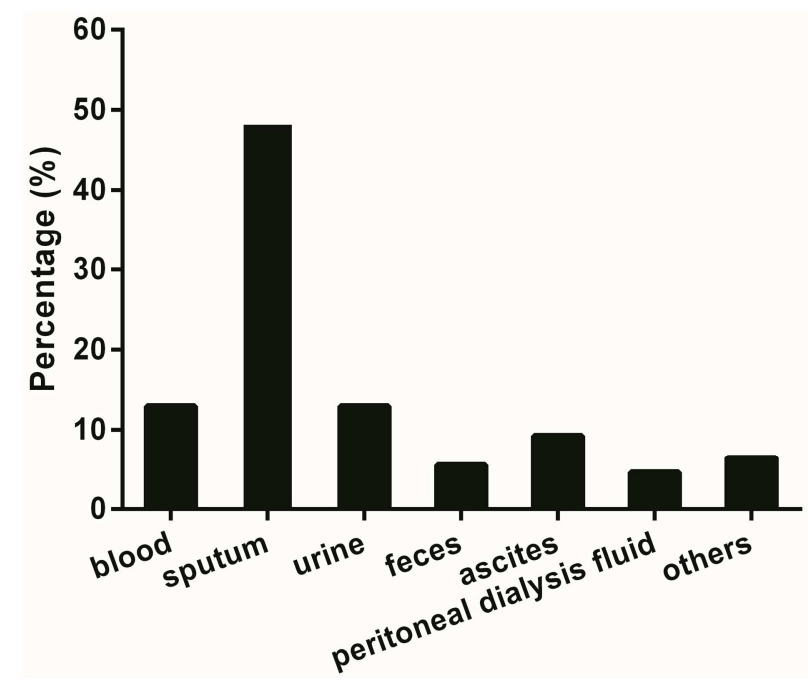

Figure 2. Specimen distributions of IFI.

\subsection{Comparison of $G$ Test and Fungal Culture}

The positive rate of $\mathrm{G}$ test was $39.95 \%$, higher than that of fungus culture $(28.95 \%, \mathrm{P}<0.001)$. And the positive rate of combined $\mathrm{G}$ test and fungal culture was $43.70 \%$. The $\kappa$ value of $\mathrm{G}$ test and fungal culture was $0.596(\mathrm{P}<0.001)$, indicating moderate consistency.

When positive fungal culture was used as the criterion for the diagnosis of IFI, the sensitivity, specificity, positive predictive value and negative predictive value of $\mathrm{G}$ test for the diagnosis of IFI were $87.04 \%, 79.25 \%, 63.09 \%$ and $93.75 \%$, respectively.

When positive $G$ test was used as the criterion for the diagnosis of IFI, The sensitivity, specificity, positive predictive value and negative predictive value of IFI in fungal culture diagnosis were $63.09 \%, 93.75 \%, 87.04 \%$ and $79.25 \%$, respectively (Table 1 ).

Table 1. Diagnosis of IFI by $G$ test and fungal culture.

\begin{tabular}{lllll}
\hline & & \multicolumn{2}{l}{ fungal culture } & \multirow{2}{*}{ total } \\
\cline { 3 - 4 } & positive & negative & \\
\hline \multirow{3}{*}{ G test } & positive & 94 & 55 & 149 \\
& negative & 14 & 210 & 224 \\
& total & 108 & 265 & 373 \\
\hline
\end{tabular}

\subsection{Comparison of Therapeutic Effects}

All 373 suspected IFI patients were treated with antifungal therapy, and were grouped according to the results of $G$ test and fungal culture. The effective rate of the both methods positive group was higher than that in the only fungal culture positive group $(72.34 \%$ vs $42.86 \%, \mathrm{P}=0.033)$ and the both methods negative group $(72.34 \%$ vs $30.48 \%, \mathrm{P}<0.001)$. The effective rate of the only $\mathrm{G}$ test positive group was $58.18 \%$, higher than that of the both methods negative group
$(\mathrm{P}<0.001)$. There was no significant difference in the therapeutic efficiency between the only $\mathrm{G}$ test positive group and the only fungal culture positive group $(\mathrm{P}=0.303$, Table 2 , Figure 3).

Table 2. The therapeutic effect of IFI.

\begin{tabular}{llll}
\hline & effective & ineffective & total \\
\hline G test and fungal culture positive & $68(72.34 \%)$ & $26(27.66 \%)$ & 94 \\
only G test positive & $32(58.18 \%)$ & $23(41.82 \%)$ & 55 \\
only fungal culture positive & $6(42.86 \%)$ & $8(57.14 \%)$ & 14 \\
G test and fungal culture negative & $64(30.48 \%)$ & $146(69.52 \%)$ & 210 \\
total & $170(45.58 \%)$ & $203(54.42 \%)$ & 373 \\
\hline
\end{tabular}

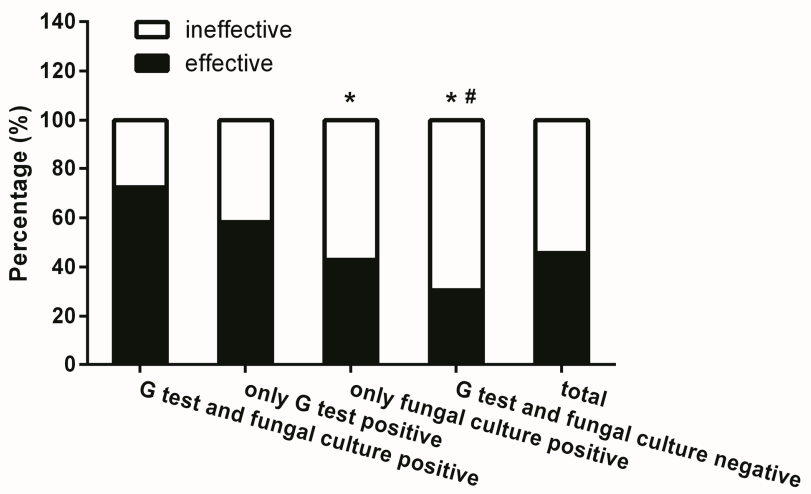

Figure 3. Comparison of the therapeutic effect of IFI (*indicated comparison with $G$ test and fungal culture positive group, $p<0.05$; \#indicated comparison with only $G$ test positive group, $p<0.05$ ).

\section{Discussion}

Early diagnosis was crucial to IFI, so a rapid and effective non-invasive detection method was needed in clinical practice. This study showed that among 373 suspected IFI patients, the positive rate of $\mathrm{G}$ experiment was $39.95 \%$, higher than that of fungus culture $(28.95 \%, \mathrm{P}<0.001)$. The positive rate of combined $\mathrm{G}$ test and fungal culture was $43.70 \%$. The $\kappa$ value of $\mathrm{G}$ test and fungal culture was $0.596(\mathrm{P}<0.001)$, indicating moderate consistency. When positive fungal culture was used as the criterion for the diagnosis of IFI, the sensitivity, specificity, positive predictive value and negative predictive value of $\mathrm{G}$ test for the diagnosis of IFI were $87.04 \%, 79.25 \%$, $63.09 \%$ and $93.75 \%$, respectively. The effective rate of the both methods positive group was $72.34 \%$, higher than $42.86 \%$ in the only fungal culture positive group $(\mathrm{P}=0.033)$ and $30.48 \%$ in the both methods negative group $(\mathrm{P}<0.001)$. The effective rate of the only $\mathrm{G}$ test positive group was $58.18 \%$, higher than that of the both methods negative group $(30.48 \%, \mathrm{P}<0.001)$. There was no significant difference in the therapeutic efficiency between the only $\mathrm{G}$ test positive group and the only fungal culture positive group $(\mathrm{P}=0.303)$.

The incidence of IFI was increasing year by year, and the mortality rate remained high despite vigorous antifungal treatment. One of the important reasons was the difficulty in early diagnosis of deep fungal infection, which delayed the clinical anti-fungal treatment, worsened the patient's condition and increased the mortality rate [8]. Therefore, early diagnosis 
played an important role in the treatment and prognosis of IFI [9]. Clinical diagnosis of IFI mainly consisted of four parts: host factors (preventive treatment), clinical characteristics (empirical treatment), microbiological examination (preemptive treatment) and histopathology (pathogen treatment) [7]. Histopathological examination was the "gold standard" for the diagnosis of IFI, but as an invasive examination, it had been restricted in clinical practice [10]. Compared with the time-consuming and low detection rate of fungal culture [11], as a unique microbiological detection method for fungal infection [12], $\mathrm{G}$ test had the advantages of being specific, fast and accurate.

(1-3)- $\beta$-D-glucan was a polysaccharide component of the cell walls of fungi, specific to fungi other than zygomycetes and Cryptococcus [13]. Glucan was absent from prokaryotes, bacteria, viruses and human cells, but was widely present in the cell walls of fungi. When the fungus entered the blood or deep tissue of the human body, it could be released from the cell wall by phagocytosis and digestion of phagocytic cells, thus increasing the level of (1-3)- $\beta$-D-glucan in blood and other body fluids (such as urine, cerebrospinal fluid, peritoneal effusion, pleural effusion, etc.). When the fungus was killed, the immune system quickly cleared (1-3)- $\beta$-D-glucan. However, the increase of plasma (1-3)- $\beta$-D-glucan was not obvious during superficial fungal infection or fungal colonization [14]. Elevated levels of (1-3)- $\beta$-D-glucan in blood and other sterile body fluids indicated IFI, most commonly caused by aspergillus and candida. Therefore, $G$ test [(1-3)- $\beta$-D-glucan assay] in the blood of patients can be used as an early predictor of IFI [10, $15,16]$. In this study, when positive fungal culture was used as the criterion for the diagnosis of IFI, the sensitivity, specificity, positive predictive value and negative predictive value of $\mathrm{G}$ test for the diagnosis of IFI were $87.04 \%, 79.25 \%, 63.09 \%$ and $93.75 \%$, respectively. The results showed that $\mathrm{G}$ test had higher sensitivity and negative predictive value, suggesting better diagnostic value and negative exclusion ability.

Pathogenic microbiology allowed IFI to preempt treatment. In terms of therapeutic efficacy, the results showed that the effective rate of both methods positive group was as high as $72.34 \%$, and the effective rate in the only $\mathrm{G}$ test or only fungal culture positive group was $58.18 \%$ and $42.86 \%$, respectively. $\mathrm{G}$ test only needed about $2 \mathrm{~h}$ to issue a report, saving time for clinicians to intervene as early as possible; and fungal culture could clearly identify the infected bacteria and conduct in vitro drug susceptibility test, providing a favorable basis for clinical accurate drug use. Therefore, combining these two methods could effectively increase the diagnosis rate and treatment efficiency of IFI. Further, the negative predictive value of $\mathrm{G}$ test in the diagnosis of IFI was $93.75 \%$, whose negative result was helpful to exclude IFI. It was very important to guide clinical rational drug use and prevent the overuse of antifungal drugs. Therefore, the $\mathrm{G}$ test had great value for the early diagnosis and treatment of IFI.

This study had some advantages and limitations. First of all, we conducted a comprehensive analysis of the microbiological test results and clinical treatment efficacy of
373 patients with IFI, and evaluated the diagnostic value of $G$ test and fungal culture. Secondly, G test, as a non-invasive and efficient index, had a high predictive value for IFI and was suitable for clinical application. Limitations included the following points: the sample size was relatively small; due to only one center was included, multicenter data and follow-up studies are necessary; and lack of histopathology data, which was the gold standard.

\section{Conclusion}

With the increasing incidence of IFI, we need to pay attention to its early diagnosis and treatment. $G$ test is a non-invasive and effective method in the diagnosis of IFI. Combined with fungal culture, it can improve its positive rate and has a higher guiding value for clinical medication, which is worthy of clinical promotion.

\section{Competing Interests}

The authors declare that they have no competing interests.

\section{Acknowledgements}

The authors would like to acknowledge all patients and their families for participating in this study.

Funding: This work was financially supported by the research project of Guangdong Provincial Bureau of traditional Chinese Medicine, China (No. 20191089).

\section{References}

[1] Mikulska, M., E. Furfaro and C. Viscoli, Non-cultural methods for the diagnosis of invasive fungal disease. Expert Rev Anti Infect Ther, 2015. 13(1): p. 103-17.

[2] Groll, A. H. and J. Ritter, [Diagnosis and management of fungal infections and pneumocystis pneumonitis in pediatric cancer patients]. Klin Padiatr, 2005. 217 Suppl 1: p. S37-66.

[3] Denning, D. W. and P. Munoz, Advances in invasive fungal infection and antifungal therapy: Introduction. Clin Microbiol Infect, 2001. 7 Suppl 2: p. vi.

[4] Pickering, J. W., et al., Evaluation of a (1-\&gt;3)-beta-D-glucan assay for diagnosis of invasive fungal infections. J Clin Microbiol, 2005. 43(12): p. 5957-62.

[5] Schuetz, A. N., Invasive fungal infections: biomarkers and molecular approaches to diagnosis. Clinics in laboratory medicine, 2013. 33(3): p. 505.

[6] Montagna, M. T., et al., Epidemiology of invasive fungal infections in the intensive care unit: results of a multicenter Italian survey (AURORA Project). Infection, 2013. 41(3): p. 645-53.

[7] Kontoyiannis, D. P., A clinical perspective for the management of invasive fungal infections: focus on IDSA guidelines. Infectious Diseases Society of America. Pharmacotherapy, 2001. 21(8 Pt 2): p. 175S-187S. 
[8] Murri, R., et al., Performance evaluation of the (1, 3)-beta-D-glucan detection assay in non-intensive care unit adult patients. Infect Drug Resist, 2019. 12: p. 19-24.

[9] Yoshida, K., H. Shoji and T. Takuma, Clinical viability of fungitell, a new (1-3)- $\beta$-D-glucan measurement kit, for diagnosis of invasive fungal infection, and comparison with other kits available in Japan. J Infect Chemother., 2011. 17(4): p. 473-477.

[10] Giacobbe, D. R., et al., Combined use of serum (1, 3)-beta-D-glucan and procalcitonin for the early differential diagnosis between candidaemia and bacteraemia in intensive care units. Crit Care, 2017. 21(1): p. 176.

[11] He S, et al., A systematic review and meta-analysis of diagnostic accuracy of serum 1, 3- $\beta$-D-glucan for invasive fungal infection: Focus on cutoff levels. J Microbiol Immunol Infect., 2014. 48(4): p. 351-361.

[12] Shabaan AE, E. L. E. W., Role of serum $(1,3)-\beta$-d-glucan assay in early diagnosis of invasive fungal infections in a neonatal intensive care unit. J Pediatr (Rio J). 2018 Sep - Oct; 94(5):559-565.

[13] Nucci, M., et al., Performance of 1, 3-beta-D-glucan in the diagnosis and monitoring of invasive fusariosis. Mycoses, 2019.

[14] Hachem, R. Y., et al., Utility of galactomannan enzyme immunoassay and $(1,3)$ beta-D-glucan in diagnosis of invasive fungal infections: low sensitivity for Aspergillus fumigatus infection in hematologic malignancy patients. J Clin Microbiol, 2009. 47(1): p. 129-33.

[15] Tran T, B. S., Application of the 1, 3-b-D-Glucan (Fungitell) Assay in the Diagnosis of Invasive Fungal Infections. Arch Pathol Lab Med. 2016 Feb; 140(2):181-5..

[16] Trager, J., et al., Serum (1--\&gt;3)-beta-D-glucan and galactomannan levels in patients with cystic fibrosis: a retrospective cohort study. BMC Pulm Med, 2018. 18(1): p. 52. 